\section{The Complicated Equation of Smell, Flavor, and Taste}

\author{
M. Castillo, Editor-in-Chief
}

O ur oldest senses are those related to chemogustatory capacities: smell and taste. Of these, smell is probably the oldest, and before we fully develop cerebral hemispheres, the olfactory apparatus already exists as extensions of the limbic system. The study of the senses of smell and taste is so complex that it encompasses armies of aromachologists, food scientists, physiologists, behavioral psychologists, cognitive neuroscientists, neuropharmacologists, biochemists, anthropologists, molecular biologists, and many more and is intimately related to the study of taste.

The olfactory system in vertebrates has a unique embryology. It forms from 1) paired placodes made of non-neural epithelium that have the capacity to give rise to sensory neurons and supporting cells in the olfactory epithelium, and 2) neural crest cells that give origin to the structural elements of the nose and its cavities. ${ }^{1}$ Although one cannot form without the other, neural crest cells get to their destination first. The olfactory receptor neurons are in the nasal cavity, and their axons, arranged in fascicles, traverse the cribriform plates and dura to synapse with cells in the olfactory bulbs, which are extensions of the brain. The olfactory neurons and accompanying glial cells arise outside the central nervous system but have the capacity to regenerate throughout life; it seems that progenitor neural crest cells may be their origin.

The human sense of smell is bidirectional, and the way we perceive smells changes according to the direction of air flow. Orthonasal smell is perceived when breathing in, while retronasal smell occurs when odorized air arising from the mouth is forced into the nose. This last type of smell is much more complex than the first one because it recruits flavor, texture, hearing, and muscle activity. Animals with a great sense of smell like dogs are designed predominantly for orthonasal smell. Their long snouts concentrate, moisturize, and direct odorized air directly toward their olfactory epithelium, assuring that warmed molecules are easily detected. Dogs have over 220 million olfactory receptors (compared with 5-10 million in humans), a 40\% greater area of the brain dedicated to smell, and the ability to smell 1000-10,000 times better than humans. ${ }^{2}$ In addition, dogs have a large vomeronasal (Jacobson) organ, whose neurons extend to accessory olfactory bulbs and then to the hippocampi. This organ is predominantly involved in pheromone perception and does not play an important role in the human sense of smell. Pheromones have a "blind smell," meaning that they stimulate the brain (observed with fMRI) while having no odor that can be perceived. Females are sensitive to male pheromones, particularly during ovulation. Male pheromones are found in sweat, but only fresh sweat. After 20 minutes, sweat is oxidized and it just smells bad. Billing agencies will send out bills scented with androstenone (a pheromone)

http://dx.doi.org/10.3174/ajnr.A3739 because they are then perceived as being more aggressive and increase their collection rates ${ }^{3}$ (note: the report in this reference is wonderfully entertaining!).

Because humans mostly depend on vision that is stereoscopic, which in turn is contingent on a strict interocular distance, we do not have a long snout and our anterior nasal pathways are less complex, less efficient, and shorter than those of dogs. However, contrary to prior beliefs, there is no evolutionary competition between smell and taste and vision; our sight has improved but our sense of smell remains quite good and the blind do not have a better sense of smell than the sighted. Although most mammals depend on orthonasal smell, we humans mostly use retronasal smell. When we say something tastes good, in reality we mean that it smells good because most "flavor" is actually retronasal smell. While retronasal smell is essential for tasting, antegrade smelling is not.

Once odorized air enters the nose in antegrade or retrograde directions, it reaches the cilia of the olfactory neurons where about 1000 specific receptor proteins are present. Specific olfactory receptor genes encode each protein. The discoverers of these genes were awarded the 2004 Nobel Prize. ${ }^{4}$ Because humans can see very well, we do not depend on smell too much. The development of tricolor vision led to many olfactory receptor genes disappearing. ${ }^{5}$ Humans can still differentiate about 10,000 smells, but to name them all, you have to be an expert such as wine connoisseur Robert Parker. The molecules for each smell have individual chemical and physical configurations that allow them to bind with specific receptors (the so-called "lock and key" concept). Once the molecules bind, adenylate cyclase is stimulated, and the result is an electrical impulse carried to the mitral cells that reside in the olfactory bulbs and send axons to different parts of the brain as follows:

- The piriform cortex is formed by the amygdala, uncus, and parahippocampal gyrus and is involved in perception of smells.

- The entorhinal cortex is the anterior aspect of the parahippocampal gyrus. Its function is to pair specific odors to specific memories (remember that an abnormal sense of smell is typical of Alzheimer and Parkinson disease). ${ }^{6,7}$

- The olfactory tubercle is located close to the nucleus accumbens; it is not directly involved in the perception of smells but rather in reward behaviors associated with odors.

- The amygdala is involved in emotional and autonomic responses to odors.

A good sense of smell can make up for the loss of taste as seen in the case of Chef Grant Achatz. Mr Achatz's Chicago restaurant, Alinea, now ranks as the sixth best in the world (it also has been awarded 3 Michelin Stars). Mr Achatz developed and neglected an oral cancer until it became stage 4 , and rather than losing his tongue, he decided to undergo chemotherapy, radiation, and surgery. ${ }^{8}$ Although his cancer is in remission, he lost his sense of taste (but not his sense of smell). I have eaten at Alinea and can assure our readers that this handicap has not affected the taste of the wonderful food he there designs and prepares. The relationship between smell, taste, and the brain is studied by a discipline called "neurogastronomy."

Smells produce activation in specific olfactory bulb regions, depending on their specific chemical compounds and timing. It 
seems that olfactory bulbs use mechanisms similar to our "visual pattern recognition" to identify discrete smells. "Odor images" refer to maps of olfactory bulb activity during olfaction. The SenseLab Web site contains many of these maps obtained with fMRI at 7T in animals (because the olfactory bulb contains neurons, their activation may be mapped by fMRI as we commonly do in the brain). ${ }^{9}$ Curiously, smell perception may occur after all related brain areas are damaged, as long as the olfactory bulbs remain intact.

Similar to the olfactory system, taste depends on the specific recognition of different flavors by specific cells in the tongue. We Westerners recognize 4 stimuli (salt, acid, sweet, and bitter), while Asians can add Umami (savory or meatlike) to these. Unlike the olfactory cells, taste does not get to the brain directly but via the seventh, ninth, and tenth cranial nerves. It is unclear whether stimuli traveling through these nerves compete and complement each other, but the end of the road is the insular cortex, where taste becomes a conscious activity. Weak smells and taste are congruent with each other, and they add up to recognizable flavors. Molecular cuisine, like that practiced in Alinea, El Bulli, and other famous modernistic restaurants, is based on the combination of noncongruent ingredients that result in a new perception of flavors. For example, Adam Melonas, a disciple of El Bulli's Chef Ferran Adria, created the "Octopop," a lollipop made of sliced, orange-infused octopus. ${ }^{10}$ In this dish, the strong retronasal smell of oranges mixed with the texture of the octopus leads to sensory fusion and a greater recruitment of brain regions needed to analyze what is in one's mouth, resulting in a totally new experience.

Chefs can only go so far because most tastes and smells have strong emotional (hedonic) components that may render some combinations repellent. Last year, my wife and I decided to eat at Corton's in New York and found ourselves disliking their utterly strange combination of flavors. Sensory fusion overload did us in, but what we disliked may have been pleasurable to others, particularly individuals (supertasters) who may be able to taste the individual components in a dish and enjoy them for what they are. When aiming for sensory fusion, one needs exact amounts of ingredients (that is why molecular cuisine is considered very close to chemistry). For example, add too much capsaicin and this irritant suppresses the taste of all else in a bite.

Although the title of this Perspectives refers to the equation "smell + taste = flavor," one must also add "mouth sense" to make it correct. Mouth sense is important to taste and refers to temperature, pain, touch, and pressure receptors inside the oral cavity. These sensations are transmitted to the somatosensory cortex via the trigeminal nerves. The cornerstones of molecular (also called modernistic) cuisine-foams, spheres, and powders-rely on producing a flavor with a totally different mouth sense to surprise us. Next, we must add vision to the equation. Vividly colored food tastes and smells more intense than bland colored food. ${ }^{a}$ Conditioning also plays a role, and

\footnotetext{
a "Synesthesia" refers to an ability to see color or hear sounds when smelling particular odors. Some individuals are gifted and have a broad sense of synesthesia (such as perfume makers); however, all of us have some synesthetic ability.
}

if, for example, white wines are colored red, many tasters will believe that they were given red wines. The last addition to the equation is hearing. If we buy a cracker said to be "crispy," it must produce a sound of more than $5 \mathrm{kHz}$ in our mouth for us to perceive it as such. Carrots, which we expect to be "crunchy," produce a sound between 1 and $2 \mathrm{kHz}$.

In his book Neurogastronomy, ${ }^{11}$ Gordon M. Shepherd, a professor of neurobiology at Yale University, refers to the "flavor perception system." ${ }^{\text {b }}$ Professor Shepherd calls the other half of the equation (emotion + memory + decisions + plasticity + language + consciousness $=$ flavor) the "flavor action system," and one system will not work without the other. Scientists who study addictions (particularly drug and food ones), as well as neuroeconomists, are very interested in these systems. On the basis of understanding both, the food industry has created the most addictive and universal food item: the French fry (buttery smell, salty taste, crunchy feel and sound, and a vivid yellow color). The industry also knows that children prefer sweet and salty over sour and bitter. Colors do strange things to flavor and our desire to eat. Orange and yellow are said to induce appetite and thus are used in the décor of many fast food restaurants. Green, brown, and red are the most used colors in the food industry because they are the naturally occurring ones and we associate them with nature and thus, health. Blue is linked to sweetness, but it is not a natural food color and we tend to avoid food of this color. Colors and taste have no relationship with nutritional value. Smells cause strange sensations; who would have imagined that the smell of pumpkin pie increases penile blood flow?

As we age; our senses of smell and taste deteriorate, a finding nearly universal after 60 years of age, and we are all familiar with the consequences of adding too much salt or sugar to our food. By 80 years of age, most individuals' sense of smell is significantly impaired (though women fare much better than men). Cuisine for the elderly attempts to compensate for decreasing senses by making food more palatable. Regardless of age, the flavor equation is complex, and I like to think of it as follows:

$($ smell + taste + mouth sense + sight + sound $) \times$ (emotion + memory + decisions + plasticity + language + consciousness) $=$ flavor.

\section{REFERENCES}

1. Katoh H, Shibata S, Fukuda K, et al. The dual origin of the peripheral olfactory system: placode and neural crest. Molecular Brain 2011;4:34-50

2. The canine sense of smell. http://www.whole-dog-journal.com/issues/ 7_11/features/Canine-Sense-of-Smell_15668-1.html. Accessed August 14,2013

3. The smell report. http://www.sirc.org/publik/smell.pdf. Accessed August 14, 2013

4. Buck L, Axel R. A novel multigene family may encode odorant receptors: a molecular basis for odor recognition. Cell 1991;65:175-87

5. Gilad Y, Przeworski M, Lancet D, et al. Loss of olfactory receptor 
genes coincides with the acquisition of full trichromatic vision in primates. PLoS Biol 2004;2:E5

6. Wesson DW, Levy E, Nixon RA, et al. Olfactory dysfunction correlates with amyloid-beta burden in an Alzheimer's disease mouse model. J Neurosci 2010;30:505-14

7. Haehner A, Hummel T, Hummel C, et al. Olfactory loss may be a first sign of idiopathic Parkinson's disease. Mov Disord 2007;22:839-42
8. Grant Achatz. Wikipedia. http://en.wikipedia.org/wiki/Grant_ Achatz. Accessed August 14, 2013

9. Olfactory bulb odor map database. SenseLab. http://senselab.med. yale.edu/OdorMapDB. Accessed August 14, 2013

10. MADRID LAB. A progressive cuisine lab in Spain. http://www. madridlab.net/melonas. Accessed August 14, 2013

11. Shepherd GM. Neurogastronomy. New York: Columbia University Press; 2012 